APS

physics

This is the accepted manuscript made available via CHORUS. The article has been published as:

Conversion of mechanical noise into correlated photon pairs: Dynamical Casimir effect from an incoherent mechanical drive

Alessio Settineri, Vincenzo Macrì, Luigi Garziano, Omar Di Stefano, Franco Nori, and Salvatore Savasta

Phys. Rev. A 100, 022501 - Published 1 August 2019

DOI: 10.1103/PhysRevA.100.022501 


\title{
Conversion of Mechanical Noise into Correlated Photon Pairs: Dynamical Casimir effect from an incoherent mechanical drive
}

\author{
Alessio Settineri, ${ }^{1,2}$ Vincenzo Macrì ${ }^{2}$ Luigi Garziano, ${ }^{2}$ Omar Di Stefano, ${ }^{2}$ Franco Nori, ${ }^{2,3}$ and Salvatore Savasta ${ }^{1,2}$ \\ ${ }^{1}$ Dipartimento di Scienze Matematiche e Informatiche, \\ Scienze Fisiche e Scienze della Terra, Università di Messina, I-98166 Messina, Italy \\ ${ }^{2}$ Theoretical Quantum Physics Laboratory, RIKEN Cluster for Pioneering Research, Wako-shi, Saitama 351-0198, Japan \\ ${ }^{3}$ Physics Department, The University of Michigan, Ann Arbor, Michigan 48109-1040, USA
}

\begin{abstract}
We show that the dynamical Casimir effect in an optomechanical system can be achieved under incoherent mechanical pumping. We adopt a fully quantum-mechanical approach for both the cavity field and the oscillating mirror. The dynamics is then evaluated using a recently-developed master equation approach in the dressed picture, including both zero and finite temperature photonic reservoirs. This analysis shows that the dynamical Casimir effect can be observed even when the mean value of the mechanical displacement is zero. This opens up new possibilities for the experimental observation of this effect. We also calculate cavity emission spectra both in the resonant and the dispersive regimes, providing useful information on the emission process.
\end{abstract}

\section{INTRODUCTION}

One of the most surprising predictions of quantum field theory is that the vacuum of space is not empty, but it has plenty of short-lived virtual particles. Real observable particles can be produced out from the quantum vacuum providing energy to its fluctuations [1-5]. Vacuum fluctuations have measurable consequences, such as the Lamb shift of atomic spectra [6] and the modification of the electron magnetic moment [7], even when real particles are not generated. For years, scientists and researchers wondered if it was possible to achieve a direct observation of the virtual particles composing the quantum vacuum or, at least, if their conversion into real particles was achievable. The answer arrived only forty years ago, when Moore [2] suggested that a variable length cavity undergoing relativistic motion could be able to convert virtual photons into real ones. This phenomenon was later called the dynamical Casimir effect (DCE). Fulling and Davis [3] demonstrated that photons can be also generated by a single mirror subjected to a non-uniform acceleration. The DCE effect was first studied in the context of electromagnetic resonators with oscillating walls or containing a dielectric medium with time-modulated internal properties [8-11].

This concept was later generalized for other bosonic fields, e.g., cold atoms [12], phononic excitation of ion chains [13], optomechanical systems [14], and BoseEinstein condensates [15, 16]. Moreover, it has been shown that photon pairs can be emitted out from the vacuum by switching or modulating the light-matter coupling strength in cavity QED systems [17-21]. In 1996, it was shown [22] that a significant number of photons can be produced also in realistic high- $Q$ cavities with moderate mirror speeds, taking advantage of resonanceenhancement effects. Unfortunately, the resonance conditions require the mechanical frequency $\omega_{m}$ to be, at least, twice the first cavity mode frequency $\omega_{c}$, i.e., $\omega_{m} \simeq 2 n \omega_{c}$, where $n \in \mathbb{N}$. This is a significant obstacle for experimental observations.
Additional theoretical studies on the DCE have been presented in, e.g., [3, 23-29]. Some of these proposals suggested to use alternative experimental setups where the boundary conditions of the electromagnetic field are modulated by an effective motion [17, 30-35]. Specifically, the link between the DCE and superconducting circuits was theoretically proposed for the first time in Ref. [36] and elaborated later on in Ref. [37]. In this context, the experimental results did not take long to arrive. In fact, the emission of photon pairs was observed in a coplanar transmission line terminated by a SQUID whose inductance was modulated at high frequency [38]. The experimental realization of the DCE gives further evidence of the quantum nature of the dynamical Casimir radiation, indicating that the produced radiation can be strictly nonclassical with a measurable amount of intermode entanglement [39]. Reference [40] reviews vacuum amplification phenomena with superconducting circuits. Photon pairs were also produced by rapidly modulating the refractive index of a Josephson metamaterial embedded in a microwave cavity [41]. However, these do not demonstrate the conversion of mechanical energy into photon pairs, so these experiments can also be regarded as quantum simulator. A new type of optomechanical dynamical coupling based on the DCE has also been proposed in trapped Rydberg atoms interacting with a dynamical mirror whose refractive index can be periodically varied [42]. A significant emission of photon pairs has also been predicted in Mott insulators of coherently dressed three-level atoms by parametric amplification of the polaritonic zero-point fluctuations in the presence of a fast time modulation of the dressing amplitude [43].

Most theoretical studies on the DCE are based on a quantum mechanical description of the electromagnetic field and a classical description of the time-dependent boundary conditions. Recently, the DCE in cavity optomechanical systems has been investigated without linearising the dynamics and describing quantummechanically both the cavity field and the vibrating mirror [44-46]. Within this full quantum description, it 
turns out that the resonant generation of photons from the vacuum is determined by several ladders of mirrorfield vacuum Rabi-like splittings. The resulting general resonance condition for the photon pairs production is $k \omega_{m} \simeq 2 n \omega_{c},(k, n \in \mathbb{N})$. This corresponds to processes where $k$ phonons in the mechanical oscillator are converted into $n$ cavity photon pairs. This generalized resonance condition enables a resonant production of photons out from the vacuum even for mechanical frequencies lower than the lowest cavity-mode frequency, thus removing one of the major obstacles for the experimental observation of this effect.

In addition, it has been shown that a vibrating mirror prepared in an excited state (mechanical Fock state) can spontaneously emit photons like a quantum emitter. In this case, however, a photon pair is emitted instead of a single photon.

Moreover, it has been recently demonstrated that virtual Casimir photon pairs can be used to enable a coherent motional coupling between two spatially separated moveable mirrors, allowing this kind of optomechanical system to also operate as a mechanical parametric downconverter even at very weak excitations [47]. Entangled photons from the vacuum can be also generated by using microwave circuit-acoustic resonators [48].

The approach considered in Ref. [46] also extends the investigation of the DCE to the optomechanical ultrastrong-coupling (USC) regime, where the optomechanical coupling rate is comparable to the mechanical frequency [49-55]. This regime, which attracted great interest also in cavity QED giving rise to a great variety of novel quantum effects [20,56-58], turned out to be an essential feature for the realization of new interesting proposals in quantum optomechanics [59-61].

Temperature effects also play an important role for the generation of photons in a resonantly vibrating cavity [62-65]. Specifically, it turns out that the thermal contributions in these systems under the influence of time-dependent boundary conditions leads to a strong enhancement of photon pairs production at finite temperatures.

Encouraged by the results obtained in Ref. [46], here we investigate the dynamics of an optomechanical system in a fully quantum-mechanical framework, under incoherent mechanical excitation, using a master equation approach. This allows to demonstrate that a remarkable Casimir photon pairs flux is produced even considering a thermal-like noise source coupled only to the mechanical degree of freedom. For ultra-strongly coupled hybrid quantum systems [66-70], the standard quantum-optical master equation breaks down, and a dressed master equation approach is needed [56, 71, 72]. Furthermore, if the energy level spectrum displays a quasi-harmonic behaviour [51], like in optomechanical systems, a new dressed master equation $[73,74]$ not involving the usual secular approximation is required.

The outline of this article is as follows: in Sec. II we briefly introduce the theoretical model and the dressed

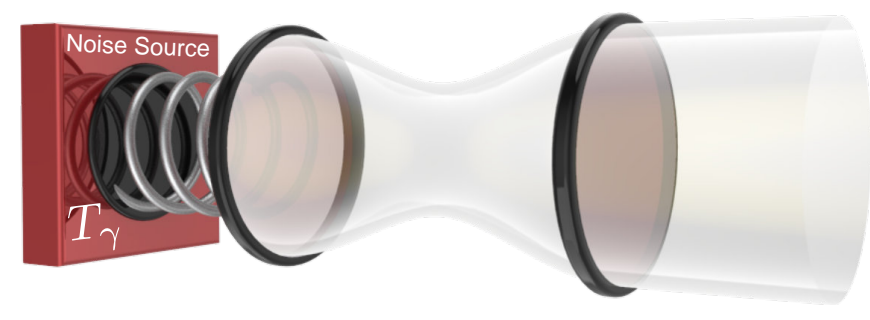

Figure 1. Schematic of a generic optomechanical system. One of the mirrors of the optical cavity is coupled to a noise source with effective temperature $T_{\gamma}$ and can vibrate at frequency $\omega_{m}$. This system can generate Casimir photon pairs.

master equation approach for quasi-harmonic hybrid systems. Section III is devoted to the presentation of the energy-level structure, focusing the attention on the avoided level crossings giving rise to the DCE. In Sec. IV we apply the generalized master equation [74] to calculate the dynamics of the system at finite temperatures and, using the quantum regression theorem, we present the power spectra in the weak and strong light-matter coupling regimes. We conclude in Sec. V.

\section{MODEL}

We study a standard optomechanical system constituted by an optical cavity with a movable end mirror [see Fig. 1]. Moreover, we consider a radiation pressure coupling between the first cavity mode and a single mechanical mode.

The system Hamiltonian can be written as:

$$
\hat{H}_{S}=\hat{H}_{0}+\hat{V}_{\mathrm{om}}+\hat{V}_{\mathrm{DCE}},
$$

where ( $\hbar=1$ throughout the paper)

$$
\hat{H}_{0}=\omega_{c} \hat{a}^{\dagger} \hat{a}+\omega_{m} \hat{b}^{\dagger} \hat{b}
$$

is the uncoupled Hamiltonian, and

$$
\hat{V}_{\text {om }}=g \hat{a}^{\dagger} \hat{a}\left(\hat{b}+\hat{b}^{\dagger}\right)
$$

is the standard optomechanical interaction Hamiltonian. Here, $\omega_{c}$ is the resonator frequency, $\omega_{m}$ is the mechanical frequency, $g$ is the optomechanical coupling strength and $\hat{a}(\hat{b}), \hat{a}^{\dagger}\left(\hat{b}^{\dagger}\right)$ are, respectively, the bosonic creation (annihilation) operators for the cavity and mechanical modes Finally, the perturbation term determining the DCE is

$$
\hat{V}_{\mathrm{DCE}}=\frac{g}{2}\left(\hat{a}^{2}+\hat{a}^{\dagger 2}\right)\left(\hat{b}+\hat{b}^{\dagger}\right) .
$$

Since in this case the $\hat{V}_{\mathrm{DCE}}$ term only couples bare states having energy differences $2 \omega_{c} \pm \omega_{m}$ much larger then the coupling strength $g$, it can be neglected. Also, this interaction term is often neglected when describing most of the experimental optomechanical systems, where the 
mechanical frequency is much smaller than the cavity frequency.

The resulting total Hamiltonian conserves the photon number and can be diagonalized separately in each $n$ photon subspace. The general quantum state of such system is:

$$
\left|n, k_{n}\right\rangle=|n\rangle \otimes \hat{D}(n \eta)|k\rangle
$$

where the integer $k_{n}$ represents the vibrational excitations of the mechanical resonator in the corresponding $n$-photon subspace, and

$$
\left|k_{n}\right\rangle=\hat{D}(n \eta)|k\rangle
$$

represents the displaced mechanical Fock state determined by the displacement operator $\hat{D}(n \eta)$, where

$$
\eta \equiv g / \omega_{m}
$$

is the normalized coupling strength. In the manifold with $n=0$, the states $\left|0, k_{0}\right\rangle$, simply labeled $|0, k\rangle$, are the eigenstates of the harmonic oscillator decoupled from the cavity. When considering ultrahigh-frequency mechanical oscillators with resonance frequencies

$$
\omega_{m} \simeq \omega_{c}
$$

the $\hat{V}_{\mathrm{DCE}}$ term cannot be neglected. In this case, the photon number is no longer conserved and there is no analytical solution for the system eigenstates. Moreover, it turns out that the introduction of the $\hat{V}_{\mathrm{DCE}}$ term increases the degree of anharmonicity, slightly modifying the levels structure but still preserving the quasi-harmonic behaviour. Consequently, the system dynamics has to be described using a generalized master equation developed without performing the usual secular approximation. A suitable approach, able to describe the time evolution of the density matrix operator $\hat{\rho}$ for any hybrid quantum system in the presence of dissipations and thermal-like noise, has been presented in Ref. [74].

In the interaction picture, this master equation can be written as

$$
\dot{\hat{\rho}}=\kappa \mathcal{L}[\hat{A}] \hat{\rho}+\gamma \mathcal{L}[\hat{B}] \hat{\rho}
$$

with $\kappa$ and $\gamma$, respectively, the cavity and mirror damping rates. The dressed photon and phonon lowering operators $\hat{O}=\hat{A}, \hat{B}$ are defined in terms of their corresponding bare operators $\hat{o}=\hat{a}, \hat{b}$ by the relation $[20,56]$

$$
\hat{O}(\omega)=\sum_{\epsilon-\epsilon^{\prime}=\omega} \hat{\Pi}(\epsilon)\left(\hat{o}+\hat{o}^{\dagger}\right) \hat{\Pi}\left(\epsilon^{\prime}\right) e^{-i \omega t}
$$

where $\epsilon$ are the eigenvalues of $\hat{H}_{S}$ and $\hat{\Pi}(\epsilon) \equiv|\epsilon\rangle\langle\epsilon|$ indicate the projectors onto the respective eigenspaces. Furthermore, the Liouvillian superoperator $\mathcal{L}[\hat{O}] \hat{\rho}$ can be ex- pressed in the general form:

$$
\begin{aligned}
\mathcal{L}[\hat{O}] \hat{\rho} & =\sum_{\left(\omega, \omega^{\prime}\right)>0} \frac{1}{2}\left\{n\left(\omega^{\prime}, T\right)\left[\hat{O}^{\dagger}\left(\omega^{\prime}\right) \hat{\rho} \hat{O}(\omega)-\hat{O}(\omega) \hat{O}^{\dagger}\left(\omega^{\prime}\right) \hat{\rho}\right]\right. \\
& +[n(\omega, T)+1]\left[\hat{O}(\omega) \hat{\rho} \hat{O}^{\dagger}\left(\omega^{\prime}\right)-\hat{O}^{\dagger}\left(\omega^{\prime}\right) \hat{O}(\omega) \hat{\rho}\right] \\
& +n(\omega, T)\left[\hat{O}^{\dagger}\left(\omega^{\prime}\right) \hat{\rho} \hat{O}(\omega)-\hat{\rho} \hat{O}(\omega) \hat{O}^{\dagger}\left(\omega^{\prime}\right)\right] \\
& \left.+\left[n\left(\omega^{\prime}, T\right)+1\right]\left[\hat{O}(\omega) \hat{\rho} \hat{O}^{\dagger}\left(\omega^{\prime}\right)-\hat{\rho} \hat{O}^{\dagger}\left(\omega^{\prime}\right) \hat{O}(\omega)\right]\right\}
\end{aligned}
$$

where $\left(k_{B}=1\right)$

$$
n(\omega, T)=[\exp (\omega / T)-1]^{-1}
$$

is the thermal noise occupation number of the system reservoir, at real or effective temperature $T$.

When counter-rotating terms are taken into account in the interaction Hamiltonian, the introduction of master equations in the dressed basis is not sufficient. Indeed, a modification of input-output relationships, relating the intracavity field with the external fields [46, 56, 74-76], is also required. According to these modified relationships, the output fields are no more determined by expectation values of the bare photon operators (see, e.g., [77-79]), but by the expectation values of the dressed operators in Eq. (10).

\section{VACUUM CASIMIR-RABI SPLITTINGS}

In order to fully characterize our system, we numerically diagonalize the Hamiltonian $\hat{H}_{S}$ in Eq. (1). Figure 2(a) shows the lowest energy levels as a function of the cavity frequency $\omega_{c} / \omega_{m}$ considering a normalized optomechanical coupling strength $\eta=0.1$.

As reported in Ref. [46], when the resonant conditions

$$
q \omega_{m}=2 \omega_{c}
$$

are satisfied, the $\hat{V}_{\mathrm{DCE}}$ term induces a coherent resonant coupling between the bare states $|0, k\rangle$ (i.e., 0 photons and $k$ phonons) and $\left|2,(k-q)_{2}\right\rangle$ (i.e., 2 photons and $k-1$ phonons), with $q \in \mathbb{N}^{*}$, having different number of excitations. Figure 2(b) shows an enlarged view of the avoided level crossing arising for $\omega_{m} \simeq \omega_{c}$, involving the states $|0,2\rangle$ and $\left|2,0_{2}\right\rangle$. When the splitting is at its minimum, the two system eigenstates are essentially a symmetric and antisymmetric linear superpositions of these bare states $\left|\psi_{ \pm}\right\rangle \simeq \frac{1}{\sqrt{2}}\left(|0,2\rangle \pm\left|2,0_{2}\right\rangle\right)$. The size of this avoided level crossing (Casimir-Rabi splitting), analytically calculated using first-order perturbation theory, is given by

$$
\begin{aligned}
2 \Omega_{0,2}^{2,0} & =2\left\langle 0,2\left|\hat{V}_{\mathrm{DCE}}\right| 2,0_{2}\right\rangle \\
& =\sqrt{2} g\left[\sqrt{3} D_{3,0}(2 \eta)+\sqrt{2} D_{1,0}(2 \eta)\right],
\end{aligned}
$$




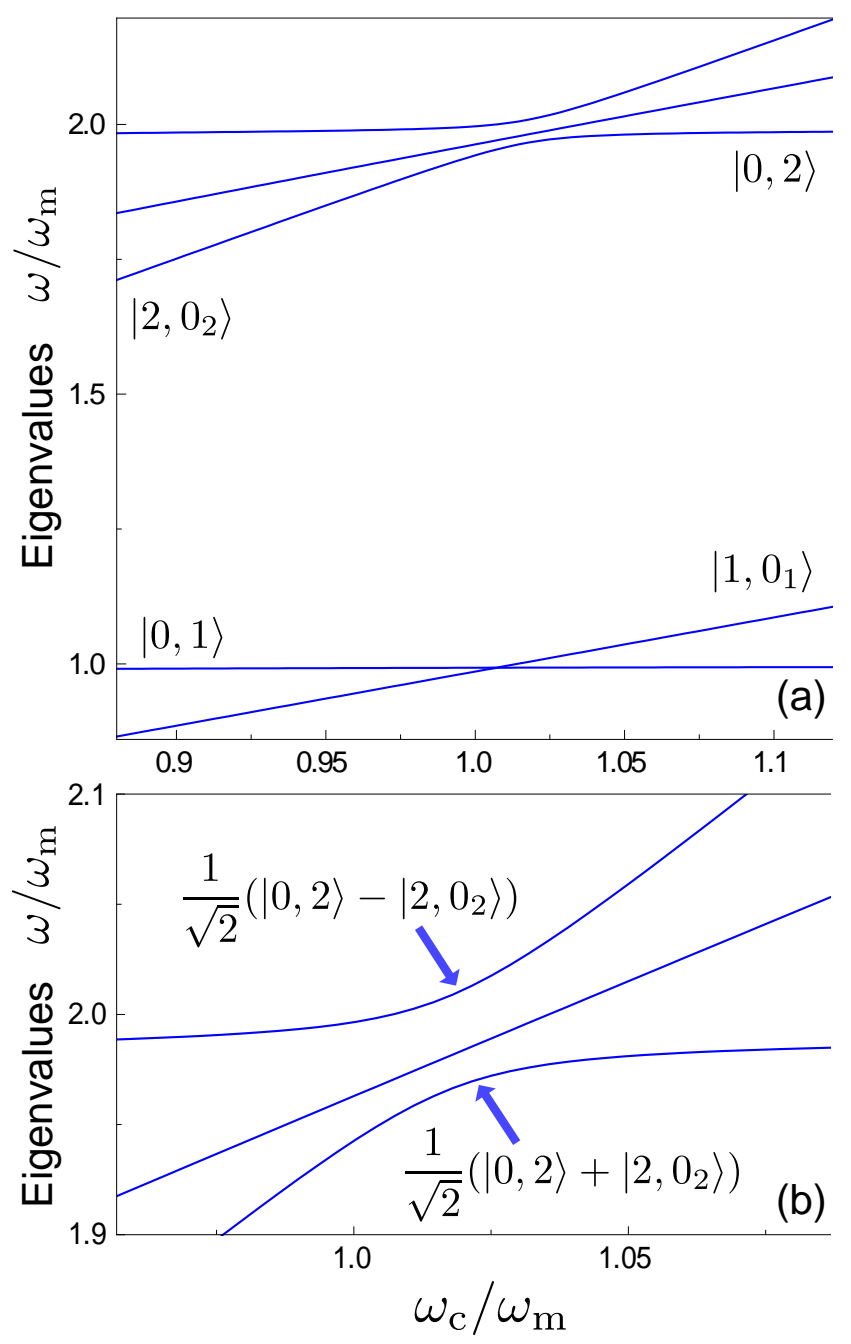

Figure 2. (a) Lowest energy eigenvalues of the system as a function of $\omega_{c} / \omega_{m}$ for a normalized optomechanical coupling strength $\eta=g / \omega_{m}=0.1$. The ground state is not displayed. (b) Enlarged view of the avoided level crossing arising from the coherent coupling between the states $|0,2\rangle$ and $\left|2,0_{2}\right\rangle$. The energy splitting reaches its minimum at the resonant frequency $\omega_{c} \simeq \omega_{m}$.

where

$$
D_{k^{\prime}, k}(2 \eta)=\sqrt{k ! / k^{\prime} !}(2 \eta)^{k^{\prime}-k} e^{-|2 \eta|^{2} / 2} L_{k}^{k^{\prime}-k}\left(|2 \eta|^{2}\right)
$$

represents the overlap between different displaced mechanical Fock states and $L_{k}^{k^{\prime}-k}$ is an associated Laguerre polynomial. It is important to note that the quantity $2 \Omega_{0,2}^{2,0}$ plays a fundamental role in the DCE, since it determines the rate at which a mechanical two-phonon state is able to generate photon pairs. Specifically, for a normalized optomechanical coupling $\eta=0.1$ we obtain a matrix element $2 \Omega_{0,2}^{2,0} \simeq 0.05$ that ensures that this avoided level crossing is able to produce a detectable rate of Casimir photon pairs.

\section{RESULTS}

Here, we present the system dynamics numerically evaluated taking into account a thermal-like pumping of the mechanical components and considering the photonic reservoir both at $T_{\kappa}=0$ and at finite temperature. Specifically, we study the time evolution of the mean phonon (photon) number $\left\langle\hat{B}^{\dagger} \hat{B}\right\rangle\left(\left\langle\hat{A}^{\dagger} \hat{A}\right\rangle\right)$ and the zero-delay phononic (photonic) normalized second-order correlation function, defined as

$$
g_{\mathrm{O}}^{(2)}(t, t)=\frac{\left\langle\hat{O}^{\dagger}(t) \hat{O}^{\dagger}(t) \hat{O}(t) \hat{O}(t)\right\rangle}{\left\langle\hat{O}^{\dagger}(t) \hat{O}(t)\right\rangle^{2}},
$$

with $\hat{O} \in[\hat{A}, \hat{B}]$.

\section{A. System dynamics in the weak-coupling regime}

We start considering the system initially prepared in its ground state and in the weak-coupling regime, which corresponds to the case where the Casimir-Rabi splitting $2 \Omega_{0, k}^{2, k-q}$ is smaller than the total decoherence rate of the system $\Gamma_{\text {tot }}=\gamma+\kappa$. Specifically, we assume $\gamma / \omega_{m}=0.05$ and $\kappa=\gamma / 2$ with an optomechanical coupling $\eta=0.1$, considering the resonant case $\omega_{m} \simeq \omega_{c}$ corresponding to the minimum splitting of the avoided level crossing arising between the states $|0,2\rangle$ and $\left|2,0_{2}\right\rangle$ (see Figure 2(b)). Figures 3(a,b) display the time evolution of the photonic $\left\langle\hat{A}^{\dagger} \hat{A}\right\rangle$ (red solid curve) and phononic $\left\langle\hat{B}^{\dagger} \hat{B}\right\rangle$ (blue dashed curve) populations, together with the time evolution of the respective two-photon and twophonon correlation functions $g_{B(A)}^{(2)}(t, t)$. All these quantities have been evaluated taking into account the interaction with a zero temperature $\left(T_{\kappa}=0\right)$ photonic reservoir and providing an incoherent thermal-like pumping of the mechanical component by means of phononic reservoir with effective temperature $T_{\gamma} / \omega_{m}=0.9$. As shown in Figure 3(a), the photonic and phononic populations start from zero and, due to the incoherent thermal-like pumping of the mechanical modes, reach a considerable stationary value. In particular, a steady state intracavity mean photon number $\left\langle\hat{A}^{\dagger} \hat{A}\right\rangle_{\mathrm{ss}} \simeq 0.15$ is obtained. For a cavity mode of frequency $\omega_{c} / 2 \pi \simeq 6 \mathrm{GHz}$, this value corresponds to a steady-state output photon flux $\Phi=\kappa\left\langle\hat{A}^{\dagger} \hat{A}\right\rangle_{\mathrm{ss}} \sim 1.4 \times 10^{8}$ photons per second. This output photon flux is remarkable since it is much higher than the detection threshold of the state-of-the-art detectors, despite the quite low quality factor $Q_{c}=\omega_{c} / \kappa=40$ of the cavity considered in the numerical calculations. Furthermore, also the mechanical loss rate $\gamma$ corresponds to a quality factor $Q_{m}$ one order of magnitude lower than the values which are experimentally measured in ultra-highfrequency mechanical resonators [80, 81]. Moreover, in Fig. 3(b) we observe that the photonic correlation function starts from a value much higher than two, suggesting that a high number of photon pairs is produced. As 

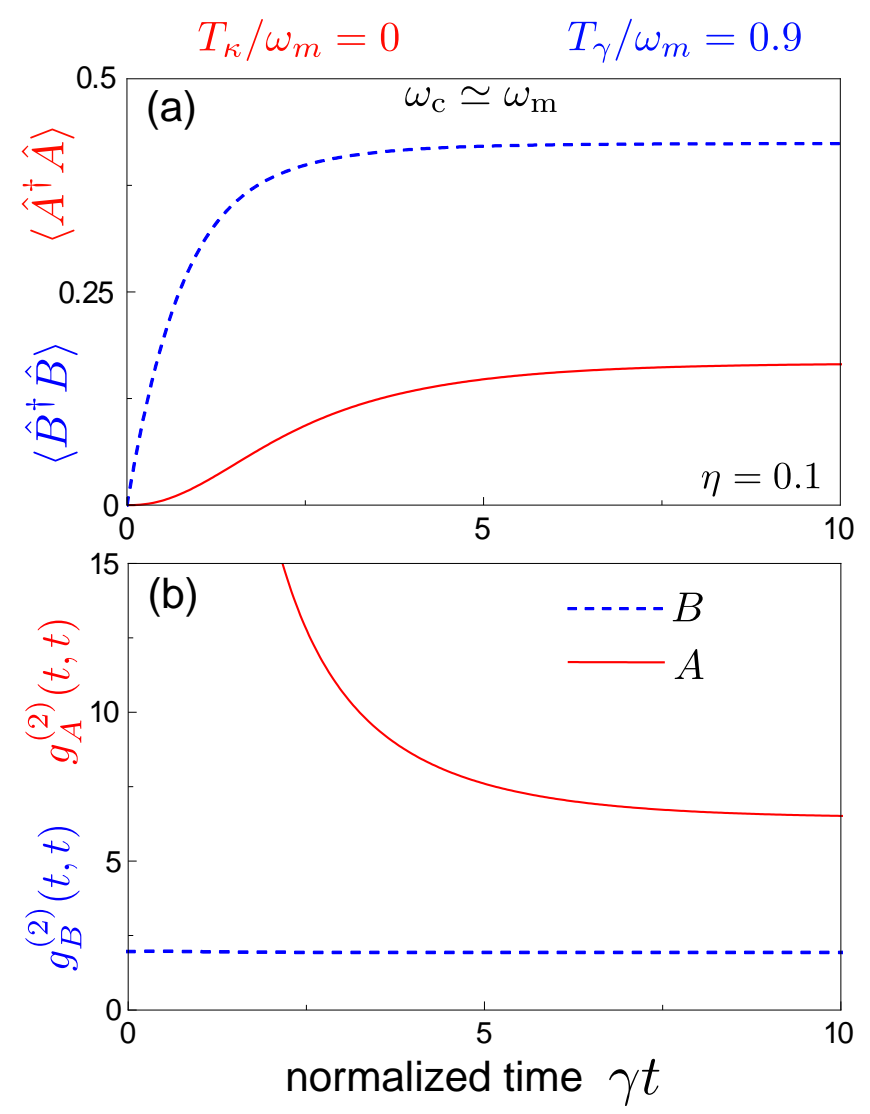

Figure 3. System dynamics for the resonant case $\omega_{c} \simeq \omega_{m}$ considering a $T_{\kappa}=0$ cavity reservoir and the mechanical oscillator coupled to a thermal-like noise source with an effective temperature $T_{\gamma} / \omega_{m}=0.9$. (a) Time evolution of the mean phonon number $\left\langle\hat{B}^{\dagger} \hat{B}\right\rangle$ (blue dashed curve) and of the mean intra-cavity photon number $\left\langle\hat{A}^{\dagger} \hat{A}\right\rangle$ (red solid curve). Due to the thermal-like pumping, the populations reach stationary values. (b) Time evolution of the zero-delay normalized photon-photon $g_{A}^{(2)}(t, t)$ and phonon-phonon $g_{B}^{(2)}(t, t)$ correlation functions. At $t=0$, the two-photon correlation function $g_{A}^{(2)}(t, t)$ displays values much higher than two, showing that a considerable number of photon pairs are emitted. As the time goes on, this value decreases significantly due to the cavity losses and the corresponding increase of the mean photon number. On the contrary, the mechanical correlation function sets on a constant value $g_{B}^{(2)}(t, t) \approx 2$, showing that the mechanical system is in an incoherent state produced by the thermal-like noise.

the time goes on, this value decreases significantly due to the system losses and the corresponding increase of the mean photon number (note that $g_{A}^{(2)}(t, t)$ is inversely proportional to the square of the mean photon number). On the contrary, the mechanical correlation function sets on a constant value $g_{B}^{(2)}(t, t) \approx 2$, showing that the mechanical component is in an incoherent state produced by the thermal-like pumping. Note that the production of Casimir photon pairs is sensitive to the optomechanical coupling strength. Figure 4 displays the temporal evolu-

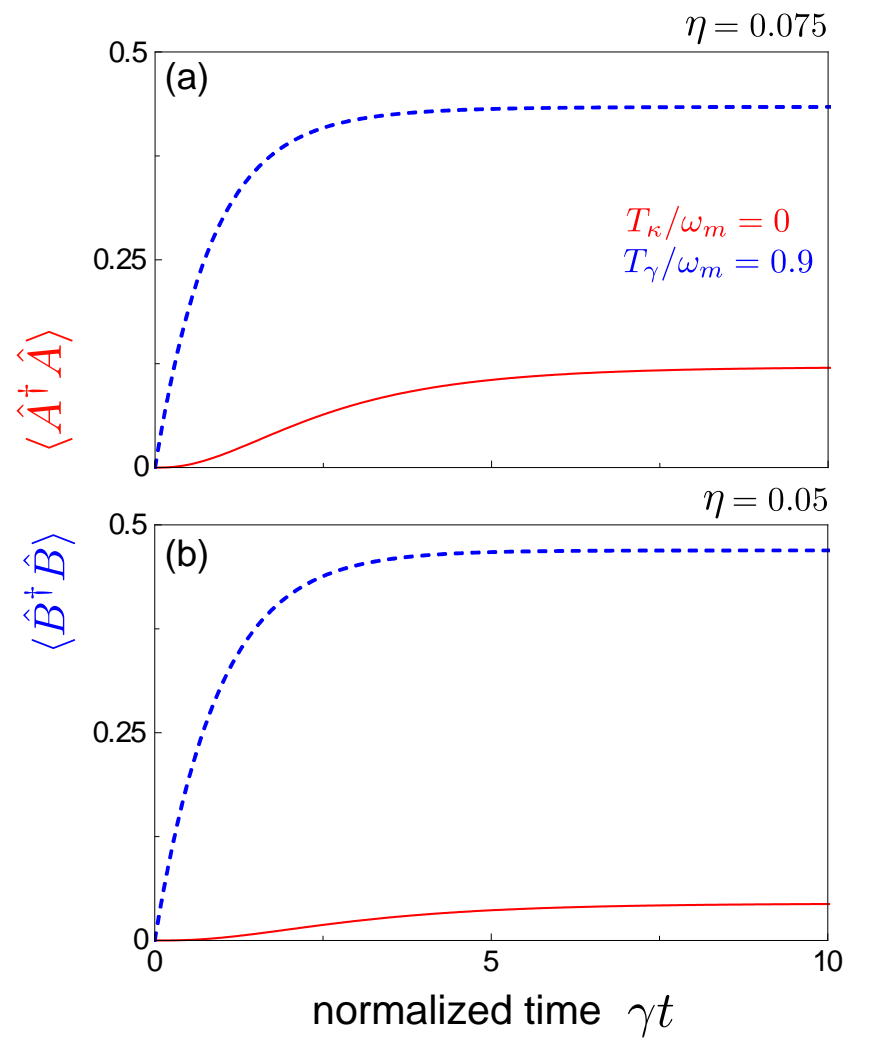

Figure 4. Time evolution of the mean phonon number $\left\langle\hat{B}^{\dagger} \hat{B}\right\rangle$ (blue dashed curve) and of the mean intra-cavity photon number $\left\langle\hat{A}^{\dagger} \hat{A}\right\rangle$ (red solid curve) for (a) $\eta=0.075$ and (b) $\eta=0.05$, in the resonant case $\omega_{c} \simeq \omega_{m}$. We consider a $T_{\kappa}=0$ cavity reservoir, while the mechanical oscillator is coupled to a thermal-like noise source with an effective temperature $T_{\gamma} / \omega_{m}=0.9$. Since the coupling rate $\Omega_{0,2}^{2,0}$ between the states $|0,2\rangle$ and $\left|2,0_{2}\right\rangle$ becomes less effective for decreasing values of $\eta$, in both cases we observe a smaller production of Casimir photon pairs with respect to the case $\eta=0.1$ displayed in Fig. 3.

tion of the photonic $\left\langle\hat{A}^{\dagger} \hat{A}\right\rangle$ (red solid curve) and phononic $\left\langle\hat{B}^{\dagger} \hat{B}\right\rangle$ (blue dashed curve) populations for (a) $\eta=0.075$ and (b) $\eta=0.05$ using the same values for the reservoir temperatures $T_{\kappa(\gamma)} / \omega_{m}$ as in Fig. 3. We observe that the intra-cavity mean photon number $\left\langle\hat{A}^{\dagger} \hat{A}\right\rangle$ decreases for decreasing values of $\eta$, suggesting that a sufficiently high optomechanical coupling strength is required in order to obtain a detectable output flux of Casimir photon pairs. This effect can be explained considering that lower values of $\eta$ lead to smaller values of the (two phonons)-(two photons) effective coupling rate $\Omega_{0,2}^{2,0}$ and, consequently, to a lower conversion rate of phonons into Casimir photon pairs. These results are particularly interesting since they demonstrate that the DCE can also be experimentally observed exciting a movable mirror with an incoherent thermal-like pump as, for example, a white noise generator (made by an ultra-high frequency resonator interacting with a microwave cavity). In real optomechanical 

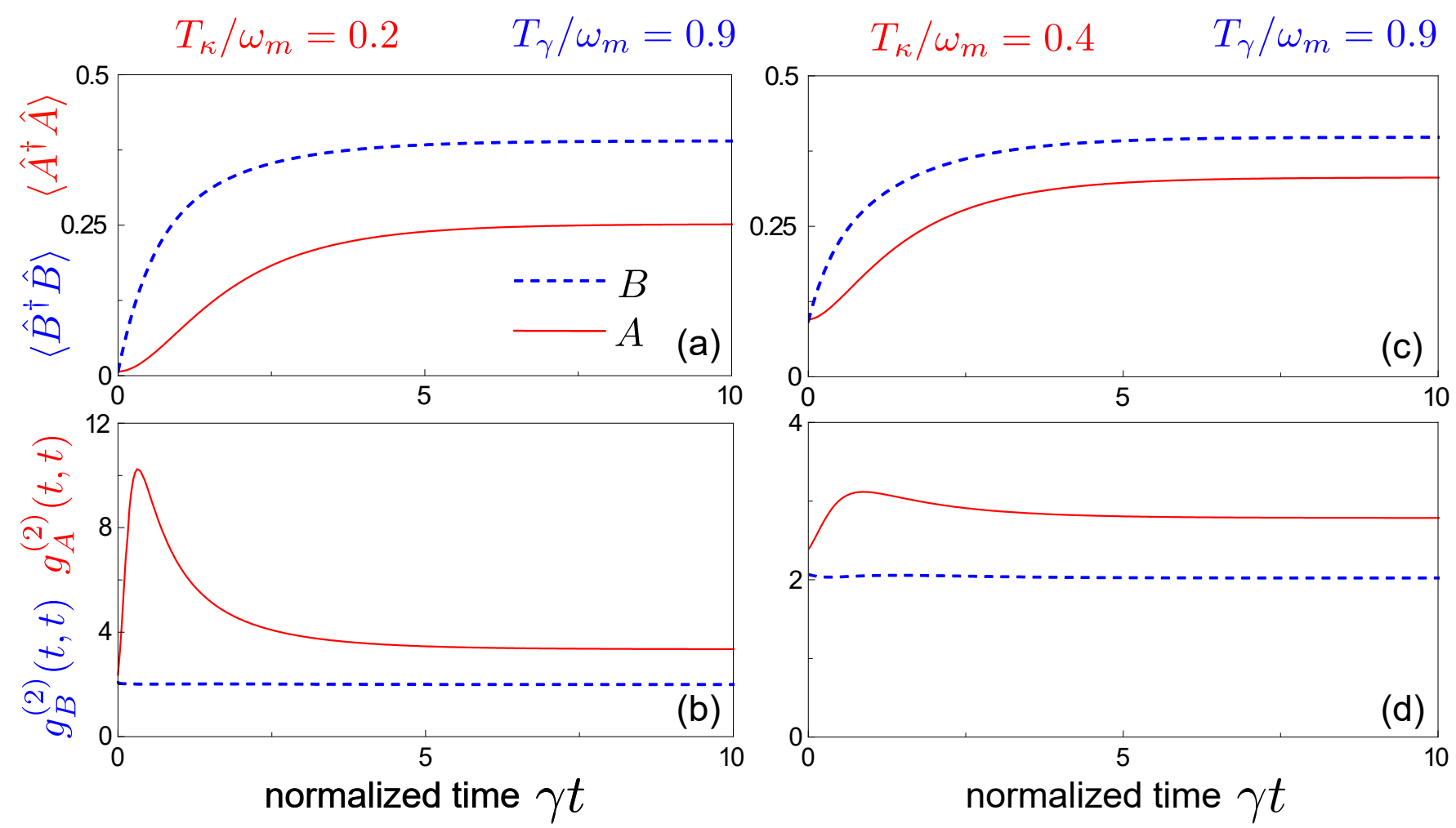

Figure 5. System dynamics evaluated considering finite-temperature reservoirs and the system initially prepared in a thermal state at the same temperature $T_{\kappa}$ of the photonic reservoir. Panels (a) and (c) show the time evolution of the cavity mean photon number $\left\langle\hat{A}^{\dagger} \hat{A}\right\rangle$ (red solid curves) and the mean phonon number $\left\langle\hat{B}^{\dagger} \hat{B}\right\rangle$ (blue dashed curves) for $T_{\gamma} / \omega_{m}=0.9$ and $T_{\kappa} / \omega_{m}=0.2(\mathrm{a}), 0.4(\mathrm{c})$. Panels (b) and (d) display the time evolution of the zero-delay two-photon (red solid curves) and two-phonon (blue dashed curves) correlation functions, $g_{A}^{(2)}(t, t)$ and $g_{B}^{(2)}(t, t)$, for $T_{\gamma} / \omega_{m}=0.9$ and $T_{\kappa} / \omega_{m}=0.2(\mathrm{~b}), 0.4(\mathrm{~d})$.

systems ground state cooling is never complete and the interaction with a finite-temperature reservoir has to be taken into account. The time evolution of the photonic and phononic populations together with the respective two-photon and two-phonon zero-delay correlation functions are displayed in Fig. 5. These functions are evaluated in more realistic conditions, taking into account non-zero temperature reservoir for both the subsystems. In these conditions, both the populations start from a non-zero value corresponding to the initial thermal equilibrium density matrix. As expected, a fraction of the observed photons is thermal and does not originate from the mechanical to optical energy conversion mechanism. This picture is confirmed by comparing the dynamics of the two correlation functions shown in Fig. 4(b,d). Specifically, when the cavity temperature increases, we observe a strong decrease of the $g_{A}^{(2)}(t, t)$ peak value, indicating that less photons are emitted in pairs. However, as expected, the phonon-phonon correlation functions remain constant at the thermal value $g_{B}^{(2)}(t, t) \simeq 2$. These results demonstrate that, when the presence of a cavity thermal noise is taken into account, the number of the produced Casimir photon pairs decrease. However, the output photon flux is still above the detection threshold of the photodetector and the peak value of the $g_{A}^{(2)}(t, t)$ indicates that photon pairs are produced.

\section{B. Emission spectra in the weak and strong coupling regimes}

In order to obtain more information on the ongoing physics, here we present the cavity emission spectra derived via a quantum regression approach. Considering a normalized optomechanical coupling $\eta=0.1$, we present results for the system both in the weak and in the strong light-matter coupling regime for different values of $\omega_{\mathrm{c}} / \omega_{m}$. We consider the cavity at $T_{\kappa}=0$, while the mechanical oscillator is coupled to a reservoir with effective temperature $T_{\gamma} / \omega_{m}=0.9$. For the sake of simplicity, we indicate the energy eigenvalues and eigenstates as $\omega_{l}$ and $|l\rangle(l=0,1, \ldots)$ and the transition frequencies as $\omega_{j k} \equiv \omega_{j}-\omega_{k}$, choosing the labeling of the states such that $\omega_{j}>\omega_{k}$, for $j>k$ [see Fig. 5(a)]. If the effective temperature of the mechanical reservoir is high enough to populate the state $|5\rangle$, the system decays toward the ground state via two different one-photon decay channels: $|5\rangle \rightarrow|2\rangle \rightarrow|0\rangle$ and $|3\rangle \rightarrow|2\rangle \rightarrow|0\rangle$. Since the states 
(a)
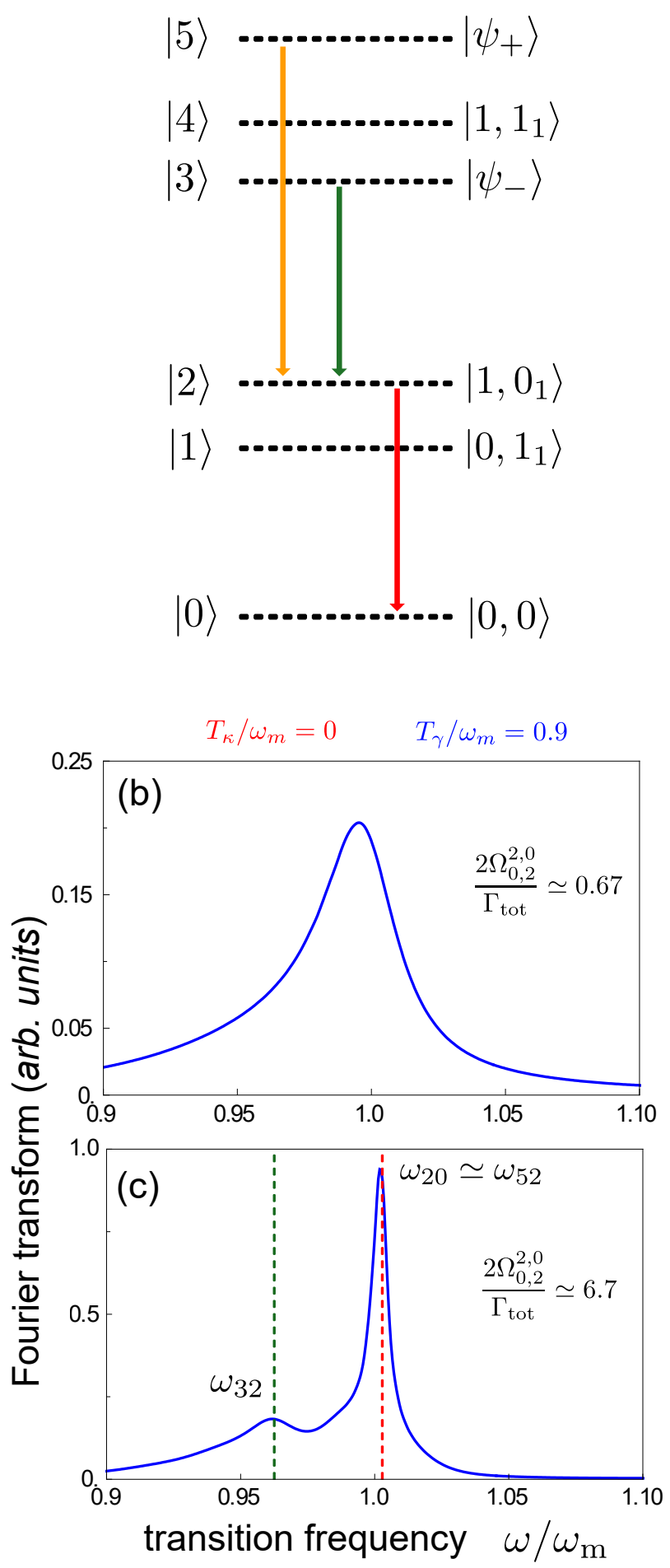

Figure 6. (a) Schematics of the first energy levels of the optomechanical system. Solid arrows represent the possible one-photon decay channels when the effective temperature of the mechanical reservoir is high enough to populate the state $|5\rangle$. Panels (b) and (c) display, respectively, the cavity emission spectra for the system in the weak and strong light-matter coupling regime and at zero detuning. In both cases, the cavity reservoir is at $T_{\kappa}=0$, while the mechanical oscillator is coupled to a reservoir with effective temperature $T_{\gamma} / \omega_{m}=0.9$. Parameters are: $\omega_{m}=1, \eta=0.1$. The total loss rate $\Gamma_{\text {tot }}=\kappa+\gamma$ of the system is: (b) $7.5 \times 10^{-2} \omega_{m}$ and (c) $7.5 \times 10^{-3} \omega_{m}$.

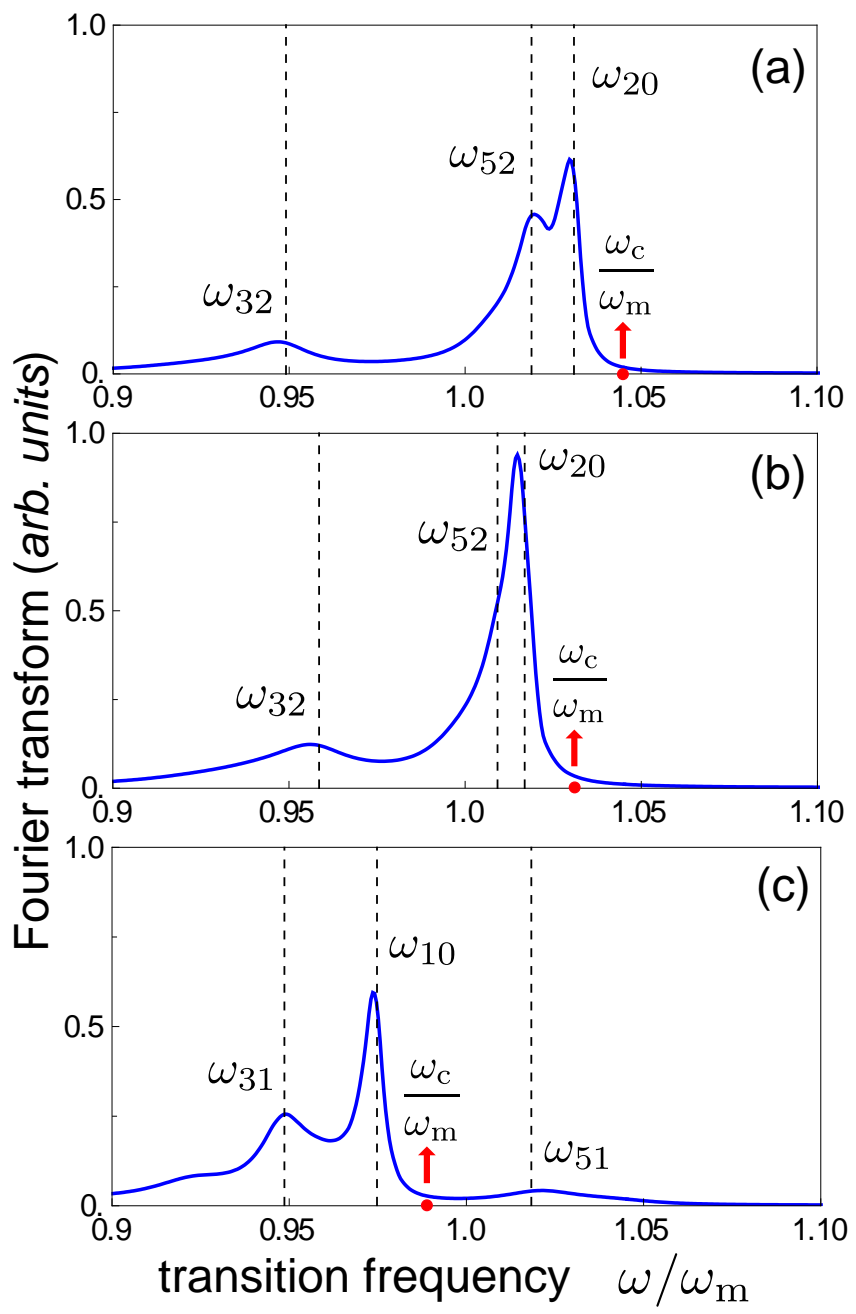

Figure 7. Cavity emission spectra for the system in the strong coupling regime for different values of the detuning $\Delta \equiv\left(\omega_{c}-\right.$ $\left.\omega_{r}\right) / \omega_{m}$, where $\omega_{r} \simeq 1.017 \omega_{m}$ is the frequency corresponding to the minimum value of the splitting in Fig. 2(b). Specifically, we considered the cases: (a) $\Delta=0.028$, (b) $\Delta=0.01$ and (c) $\Delta=-0.028$. Main contributions are indicated by dashed lines. Parameters are the same of Fig. 5(c).

$|5\rangle$ and $|3\rangle$ do not couple with the state $|4\rangle$, the other possible one-photon transition $|4\rangle \rightarrow|0\rangle$ can occur only by decays from higher energy levels.

We start considering the zero detuning case $\Delta \equiv$ $\left(\omega_{c}-\omega_{r}\right) / \omega_{m}=0$, where $\omega_{r} \simeq 1.017 \omega_{m}$ is the frequency corresponding to the minimum value of the splitting in Fig. 2(b). In this case the states $|3\rangle$ and $|5\rangle$ are well approximated, respectively, by the superpositions $\left|\psi_{ \pm}\right\rangle=\left(|0,2\rangle \pm\left|2,0_{2}\right\rangle\right) / \sqrt{2}$.

Figure 5(b) displays the emission spectra for the system in the weak coupling regime, e.g., $2 \Omega_{0,2}^{2,0}<\Gamma_{\text {tot }}$. Due to the high value of $\Gamma_{\text {tot }}$, we observe a low-resolution emission spectrum that only displays a wide band constituted by a single peak at frequency $\omega / \omega_{m} \simeq 0.98$. On the contrary, when the system is in the strong coupling regime $\left(2 \Omega_{0,2}^{2,0}>\Gamma_{\text {tot }}\right)$, the spectrum becomes well- 
resolved. As shown in Fig. 5(b), for $2 \Omega_{0,2}^{2,0} / \Gamma_{\text {tot }} \simeq 6.7$ the cavity emission spectrum displays two main peaks. Indeed, in the resonant case the accidentally quasidegenerate transitions $|5\rangle \rightarrow|2\rangle$ and $|2\rangle \rightarrow|0\rangle$ give rise to a single high-frequency peak at $\omega \simeq \omega_{m}$, whereas the lower-frequency peak at $\omega / \omega_{m} \simeq 0.98$ corresponds to the transition $|3\rangle \rightarrow|2\rangle$. It is important to notice that, in the presence of a $T_{\kappa}=0$ cavity reservoir, these peaks are observable only if the $V_{\mathrm{DCE}}$ term is included in the Hamiltonian. Indeed, without this term the states $\left|2,0_{2}\right\rangle$ and $|0,2\rangle$ are not coupled anymore and since the mechanical incoherent pumping only populates phononic states, the one-phonon decay peaks cannot be observed in the cavity emission spectra. We now turn to the numerical analysis of the detuning effects on the cavity emission spectra. Figure 6(a) displays the emission spectrum calculated for $\Delta=0.028$. As the transitions $|5\rangle \rightarrow|2\rangle$ and $|2\rangle \rightarrow|0\rangle$ are no more quasi-degenerate, the peaks at frequencies $\omega_{52}$ and $\omega_{20}$ become well resolved, while the peak corresponding to $|3\rangle \rightarrow|2\rangle$ shifts towards a slightly lower frequency. As expected, if we reduce the detuning and approach the resonance point $\Delta=0$, the spectrum essentially presents the same main features of Fig. 5(c). Specifically, Fig. 6(b) shows that for $\Delta=0.014$ the two peaks at $\omega_{52}$ and $\omega_{20}$ merge, and the emission spectrum presents only a main contibution at $\omega / \omega_{m} \simeq 1.015$, while the transition frequency $\omega_{32}$ does not change significantly. Finally, in Fig. 6(c) we study the emission spectrum in the presence of a negative detuning $\Delta=-0.028$. Also in this case, the spectrum displays three distinct peaks placed at lower frequencies respect to Fig. 6(a). This shift arises from the energy-level crossing between the states $|1\rangle$ and $|2\rangle$ shown in Fig. 2(a). Although the highest peak still corresponds to the one-photon decay toward the ground state, the emission spectrum is not symmetric respect to the one in Fig. 6(a). In particular, we observe that the intensity of the peak associated to the transition $|3\rangle \rightarrow|1\rangle$ increases, whereas the $|5\rangle \rightarrow|1\rangle$ transition peak displays a much lower intensity. This effect can be explained considering that, differently from the positive-detuning cases studied above, for $\Delta<0$ the state $|3\rangle \simeq\left|2,0_{2}\right\rangle$ has more photonic character than $|5\rangle \simeq|0,2\rangle$, which has more phononic character. Thus, while the photonic character of the polaron state $|3\rangle$ leads to an enhancement of the peak intensity at $\omega_{31}$ in the cavity emission spectrum, on the other hand the phononic character of the state $|5\rangle$ is responsible for the intensity decrease of the peak at $\omega_{51}$. This study provides useful information on the emission process. Moreover, the presence of these features in the experimental spectra would represent a signature of the production of DCE photons. A very promising experimental platform for the observation of the proposed effect is provided by circuit-optomechanical systems utilizing ultra-high-frequency $(\sim 4-6 \mathrm{GHz})$ dilatational resonators [80]. In these systems, it should be possible to easily achieve an optomechanical coupling strength $\eta=0.02$, which is rather close to the lower value considered here [see Fig. 4(b)]. Finally, we notice that a higher excita- tion noise would allow the observation of the DCE effect induced by an incoherent mechanical pumping, even for lower values of the optomechanical coupling strength.

\section{CONCLUSIONS}

In conclusion, we have studied the dynamical Casimir effect in cavity optomechanics achieved only under incoherent mechanical excitation. We employed a fully quantum-mechanical description of both the cavity field and the oscillating mirror. The system dynamics is evaluated under incoherent pumping of the mechanical component, provided by a thermal-like excitation. Using a master equation approach [74] in order to take into account losses, thermal effects and decoherence in the presence of a quasi-harmonic spectrum, we showed that a measurable flux of Casimir photons can be obtained also without a coherent pumping, suggesting another way for experimental observation of the DCE. This master equation approach could be also used to describe this effect in the presence of arbitrary colored-noise sources. The incoherent mechanical excitation mechanism described here is also expected to work in parametrically amplified optomechanical systems in order to induce two-photon hyper-Raman scattering processes, where squeezed photons already present in an optical resonator are scattered into resonant cavity-photon pairs [82]. This method would allow the parametric conversion of mechanical energy into electromagnetic energy in optomechanical systems where the mechanical frequency is usually much lower than the cavity frequency, thus eliminating the need for extremely high mechanical oscillation frequencies and ultrastrong single-photon optomechanical coupling. In Ref. [46], it has been shown that a vibrating mirror is affected by spontaneous emission, in analogy with ordinary atoms. However, it decays emitting photon pairs. Here, we show that an incoherently excited vibrating mirror can emit light, in analogy to atomic fluorescence or electroluminescence in semiconductor devices.

By applying the quantum regression theorem, we have calculated numerically the steady-state cavity emission spectra under incoherent mechanical excitation, for different detunings and loss rates. When the loss rates are lower than the effective coupling rate, the emission spectra allow to identify the different emission channels.

\section{ACKNOWLEDGMENTS}

FN is supported in part by the: MURI Center for Dynamic Magneto-Optics via the Air Force Office of Scientific Research (AFOSR) (FA9550-14-1-0040), Army Research Office (ARO) (Grant No. W911NF-18-1-0358), Asian Office of Aerospace Research and Development (AOARD) (Grant No. FA2386-18-1-4045), Japan Science and Technology Agency (JST) (via the Q-LEAP program, the ImPACT program and CREST Grant No. JP- 
MJCR1676), Japan Society for the Promotion of Science (JSPS) (JSPS-RFBR Grant No. 17-52-50023, and JSPSFWO Grant No. VS.059.18N), RIKEN-AIST Chal- lenge Research Fund, and the John Templeton Foundation. S.S. acknowledges the Army Research Office (ARO) (Grant No. W911NF1910065).
[1] J. Schwinger, "On gauge invariance and vacuum polarization," Am. J. Phys 82, 664-679 (1951).

[2] G. T. Moore, "Quantum theory of the electromagnetic field in a variable-length one-dimensional cavity," J. Math. Phys. 11, 2679 (1970).

[3] S. A. Fulling and P. C. W. Davies, "Radiation from a moving mirror in two dimensional space-time: conformal anomaly," Proc. R. Soc. A. 348, 393-414 (1976).

[4] E. Yablonovitch, "Accelerating reference frame for electromagnetic waves in a rapidly growing plasma: UnruhDavies-Fulling-DeWitt radiation and the nonadiabatic Casimir effect," Phys. Rev. Lett. 62, 1742 (1989).

[5] J. Schwinger, "Casimir light: the source," PNAS 90, 2105-2106 (1993).

[6] M. O. Scully and M. S. Zubairy, Quantum optics (Cambridge University Press, 1997).

[7] W. Greiner and S. Schramm, "Resource letter QEDV-1: the QED vacuum," Am. J. Phys 76, 509-518 (2008).

[8] V. V. Dodonov and A. B. Klimov, "Long-time asymptotics of a quantized electromagnetic field in a resonator with oscillating boundary," Phys. Lett. A 167, 309-313 (1992).

[9] V. V. Dodonov, A. B. Klimov, and D. E. Nikonov, "Quantum phenomena in nonstationary media," Phys. Rev. A 47, 4422 (1993).

[10] J.-Y. Ji, H.-H. Jung, J.-W. Park, and K.-S. Soh, "Production of photons by the parametric resonance in the dynamical Casimir effect," Phys. Rev. A 56, 4440 (1997).

[11] D. F. Mundarain and P. A. Maia Neto, "Quantum radiation in a plane cavity with moving mirrors," Phys. Rev. A 57, 1379-1390 (1998).

[12] V. V. Dodonov and J. T. Mendonça, "Dynamical Casimir effect in ultra-cold matter with a time-dependent effective charge," Phys. Scr. 2014, 014008 (2014).

[13] N. Trautmann and P. Hauke, "Quantum simulation of the dynamical Casimir effect with trapped ions," New J. Phys. 18, 043029 (2016).

[14] A. Motazedifard, M. H. Naderi, and R. Roknizadeh, "Dynamical Casimir effect of phonon excitation in the dispersive regime of cavity optomechanics," J. Opt. Soc. Am. B 34, 642-652 (2017).

[15] I. Carusotto, R. Balbinot, A. Fabbri, and A. Recati, "Density correlations and analog dynamical Casimir emission of Bogoliubov phonons in modulated atomic Bose-Einstein condensates," Eur. Phys. J. D 56, 391-404 (2010).

[16] J.-C. Jaskula, G. B. Partridge, M. Bonneau, R. Lopes, J. Ruaudel, D. Boiron, and C. I. Westbrook, "Acoustic analog to the dynamical Casimir effect in a Bose-Einstein condensate," Phys. Rev. Lett. 109, 220401 (2012).

[17] S. De Liberato, C. Ciuti, and I. Carusotto, "Quantum vacuum radiation spectra from a semiconductor microcavity with a time-modulated vacuum Rabi frequency," Phys. Rev. Lett. 98, 103602 (2007).

[18] A. A. Anappara, S. De Liberato, A. Tredicucci, C. Ciuti, G. Biasiol, L. Sorba, and F. Beltram, "Signatures of the ultrastrong light-matter coupling regime," Phys. Rev. B 79, 201303 (2009).

[19] S. De Liberato, D. Gerace, I. Carusotto, and C. Ciuti, "Extracavity quantum vacuum radiation from a single qubit," Phys. Rev. A 80, 053810 (2009).

[20] L. Garziano, A. Ridolfo, R. Stassi, O. Di Stefano, and S. Savasta, "Switching on and off of ultrastrong lightmatter interaction: photon statistics of quantum vacuum radiation," Phys. Rev. A 88, 063829 (2013).

[21] C. S. Muñoz, F. Nori, and S. De Liberato, "Resolution of superluminal signalling in non-perturbative cavity quantum electrodynamics," Nat. Commun. 9, 1924 (2018).

[22] A. Lambrecht, M.-T. Jaekel, and S. Reynaud, "Motion induced radiation from a vibrating cavity," Phys. Rev. Lett. 77, 615-618 (1996).

[23] L. H. Ford and A. Vilenkin, "Quantum radiation by moving mirrors," Phys. Rev. D 25, 2569-2575 (1982).

[24] G. Barton and C. Eberlein, "On quantum radiation from a moving body with finite refractive index," Ann. Phys. 227, 222 - 274 (1993).

[25] E. Sassaroli, Y. N. Srivastava, and A. Widom, "Photon production by the dynamical Casimir effect," Phys. Rev. A 50, 1027-1034 (1994).

[26] V. V. Dodonov and A. B. Klimov, "Generation and detection of photons in a cavity with a resonantly oscillating boundary," Phys. Rev. A 53, 2664-2682 (1996).

[27] G. Schaller, R. Schützhold, G. Plunien, and G. Soff, "Dynamical Casimir effect in a leaky cavity at finite temperature," Phys. Rev. A 66, 023812 (2002).

[28] W.-J. Kim, J. H. Brownell, and R. Onofrio, "Detectability of dissipative motion in quantum vacuum via superradiance," Phys. Rev. Lett. 96, 200402 (2006).

[29] V. V. Dodonov, "Current status of the dynamical Casimir effect," Phys. Scr. 82, 038105 (2010).

[30] Y. E. Lozovik, V. G. Tsvetus, and E. A. Vinogradov, "Femtosecond parametric excitation of electromagnetic field in a cavity," JETP Lett. 61, 723 (1995).

[31] M. Uhlmann, G. Plunien, R. Schützhold, and G. Soff, "Resonant cavity photon creation via the dynamical Casimir effect," Phys. Rev. Lett. 93, 193601 (2004).

[32] M. Crocce, D. A. R. Dalvit, F. C. Lombardo, and F. D. Mazzitelli, "Model for resonant photon creation in a cavity with time-dependent conductivity," Phys. Rev. A 70, 033811 (2004).

[33] C. Braggio, G. Bressi, G. Carugno, C. Del Noce, G. Galeazzi, A. Lombardi, A. Palmieri, G. Ruoso, and D. Zanello, "A novel experimental approach for the detection of the dynamical Casimir effect," EPL 70, 754 (2005).

[34] E. Segev, B. Abdo, O. Shtempluck, E. Buks, and B. Yurke, "Prospects of employing superconducting stripline resonators for studying the dynamical Casimir effect experimentally," Phys. Lett. A 370, 202-206 (2007).

[35] R. De Melo e Souza, F. Impens, and P. A. Maia Neto, "Microscopic dynamical Casimir effect," Phys. Rev. A 97, 
032514 (2018)

[36] J. R. Johansson, G. Johansson, C. M. Wilson, and F. Nori, "Dynamical Casimir effect in a superconducting coplanar waveguide," Phys. Rev. Lett. 103, 147003 (2009).

[37] J. R. Johansson, G. Johansson, C. M. Wilson, and F. Nori, "Dynamical Casimir effect in superconducting microwave circuits," Phys. Rev. A 82, 52509 (2010).

[38] C. M. Wilson, G. Johansson, A. Pourkabirian, M. Simoen, J. R. Johansson, T. Duty, F. Nori, and P. Delsing, "Observation of the dynamical Casimir effect in a superconducting circuit," Nature 479, 376 (2011).

[39] J. R. Johansson, G. Johansson, C. M. Wilson, P. Delsing, and F. Nori, "Nonclassical microwave radiation from the dynamical Casimir effect," Phys. Rev. A 87, 043804 (2013).

[40] P. D. Nation, J. R. Johansson, M. P. Blencowe, and F. Nori, "Colloquium: stimulating uncertainty: amplifying the quantum vacuum with superconducting circuits," Rev. Mod. Phys. 84, 1-24 (2012).

[41] P. Lähteenmäki, G. S. Paraoanu, J. Hassel, and P. J. Hakonen, "Dynamical Casimir effect in a Josephson metamaterial," Proc. Natl. Acad. Sci. USA 110, 42344238 (2013).

[42] M. Antezza, C. Braggio, G. Carugno, A. Noto, R. Passante, L. Rizzuto, G. Ruoso, and S. Spagnolo, "Optomechanical Rydberg-atom excitation via dynamic CasimirPolder coupling," Phys. Rev. Lett. 113, 023601 (2014).

[43] I. Carusotto, M. Antezza, F. Bariani, S. De Liberato, and C. Ciuti, "Optical properties of atomic mott insulators: from slow light to dynamical casimir effects," Phys. Rev. A 77, 063621 (2008).

[44] C. K. Law, "Interaction between a moving mirror and radiation pressure: a hamiltonian formulation," Phys. Rev. A 51, 2537-2541 (1995).

[45] K. Sala and T. Tufarelli, "Exploring corrections to the Optomechanical Hamiltonian," Sci. Rep. 8, 9157 (2018).

[46] V. Macrì, A. Ridolfo, O. Di Stefano, A. F. Kockum, F. Nori, and S. Savasta, "Nonperturbative dynamical Casimir effect in optomechanical systems: vacuum Casimir-Rabi splittings," Phys. Rev. X 8, 011031 (2018).

[47] O. Di Stefano, A. Settineri, V. Macrì, A. Ridolfo, R. Stassi, A. F. Kockum, S. Savasta, and F. Nori, "Interaction of mechanical oscillators mediated by the exchange of virtual photon pairs," Phys. Rev. Lett., 122, 030402 (2019).

[48] H. M. Wang, M. P. Blencowe, C. M. Wilson, and A. J. Rimberg, "Mechanically generating entangled photons from the vacuum: a microwave circuit-acoustic resonator analogue of the Unruh effect," (2018), arXiv:1811.10065.

[49] T. J. Kippenberg and K. J. Vahala, "Cavity optomechanics: back-action at the mesoscale," Science 321, 11721176 (2008).

[50] A. Nunnenkamp, K. Børkje, and S. M. Girvin, "Singlephoton optomechanics," Phys. Rev. Lett. 107, 063602 (2011).

[51] F. Marquardt and S. M. Girvin, "Optomechanics," Physics 2, 40 (2009).

[52] J. D. Teufel, D. Li, M. S. Allman, K. Cicak, A. J. Sirois, J. D. Whittaker, and R. W. Simmonds, "Circuit cavity electromechanics in the strong-coupling regime," Nature 471, 204-208 (2011).

[53] J. Chan, T. P. M. Alegre, A. H. Safavi-Naeini, J. T. Hill, A. Krause, S. Gröblacher, M. Aspelmeyer, and
O. Painter, "Laser cooling of a nanomechanical oscillator into its quantum ground state," Nature 478, 89-92 (2011).

[54] A. J. Rimberg, M. P. Blencowe, A. D. Armour, and P. D. Nation, "A cavity-Cooper pair transistor scheme for investigating quantum optomechanics in the ultra-strong coupling regime," New J. Phys. 16, 055008 (2014).

[55] M. Aspelmeyer, T. J. Kippenberg, and F. Marquardt, "Cavity optomechanics," Rev. Mod. Phys. 86, 1391-1452 (2014).

[56] A. Ridolfo, M. Leib, S. Savasta, and M. J. Hartmann, "Photon blockade in the ultrastrong coupling regime," Phys. Rev. Lett. 109, 193602 (2012).

[57] L. Garziano, R. Stassi, V. Macrì, A. F. Kockum, S. Savasta, and F. Nori, "Multiphoton quantum Rabi oscillations in ultrastrong cavity QED," Phys. Rev. A 92, 063830 (2015).

[58] L. Garziano, R. Stassi, V. Macrì, O. Di Stefano, F. Nori, and S. Savasta, "One photon can simultaneously excite two or more atoms," Phys. Rev. Lett. 117, 043601 (2016).

[59] L. Garziano, R. Stassi, V. Macrí, S. Savasta, and O. Di Stefano, "Single-step arbitrary control of mechanical quantum states in ultrastrong optomechanics," Phys. Rev. A 91, 023809 (2015).

[60] V. Macrí, L. Garziano, A. Ridolfo, O. Di Stefano, and S. Savasta, "Deterministic synthesis of mechanical NOON states in ultrastrong optomechanics," Phys. Rev. A 94, 013817 (2016).

[61] S. Butera and I. Carusotto, "Mechanical back-reaction effect of the dynamical Casimir emission," (2018), arXiv:1810.11281.

[62] G. Plunien, B. Müller, and W. Greiner, "Casimir energy at finite temperature," Physica A 145, 202 - 219 (1987).

[63] R. Schützhold, G. Plunien, and G. Soff, "Quantum radiation in external background fields," Phys. Rev. A 58, 1783-1793 (1998).

[64] G. Plunien, R. Schützhold, and G. Soff, "Dynamical Casimir effect at finite temperature," Phys. Rev. Lett. 84, 1882-1885 (2000).

[65] A. Motazedifard, A. Dalafi, M.H. Naderi, and R. Roknizadeh, "Controllable generation of photons and phonons in a coupled Bose-Einstein condensateoptomechanical cavity via the parametric dynamical Casimir effect," Ann. Physics 396, 202 - 219 (2018).

[66] O. Di Stefano, R. Stassi, L. Garziano, A. F. Kockum, S. Savasta, and F. Nori, "Feynman-diagrams approach to the quantum Rabi model for ultrastrong cavity QED: stimulated emission and reabsorption of virtual particles dressing a physical excitation," New J. Phys. 19, 053010 (2017).

[67] R. Stassi, V. Macrì, A. F. Kockum, O. Di Stefano, A. Miranowicz, S. Savasta, and F. Nori, "Quantum nonlinear optics without photons," Phys. Rev. A 96, 023818 (2017).

[68] A. F. Kockum, A. Miranowicz, V. Macrì, S. Savasta, and F. Nori, "Deterministic quantum nonlinear optics with single atoms and virtual photons," Phys. Rev. A 95, 063849 (2017).

[69] A. F. Kockum, A. Miranowicz, S. De Liberato, S. Savasta, and F. Nori, "Ultrastrong coupling between light and matter," Nat. Rev. Phys. 1, 19-40 (2019).

[70] P. Forn-Díaz, L. Lamata, E. Rico, J. Kono, and E. Solano, "Ultrastrong coupling regimes of light-matter interaction," (2018), arXiv:1804.09275.

[71] F. Beaudoin, J. M. Gambetta, and A. Blais, "Dissipation 
and ultrastrong coupling in circuit QED," Phys. Rev. A 84, 043832 (2011).

[72] D. Hu, S.-Y. Huang, J.-Q. Liao, L. Tian, and H.-S. Goan, "Quantum coherence in ultrastrong optomechanics," Phys. Rev. A 91, 013812 (2015).

[73] K. W. Ma and C. K. Law, "Three-photon resonance and adiabatic passage in the large-detuning Rabi model," Phys. Rev. A 92, 023842 (2015).

[74] A. Settineri, V. Macrí, A. Ridolfo, O. Di Stefano, A. F. Kockum, F. Nori, and S. Savasta, "Dissipation and thermal noise in hybrid quantum systems in the ultrastrongcoupling regime," Phys. Rev. A 98, 053834 (2018).

[75] S. Portolan, O. Di Stefano, S. Savasta, F. Rossi, and R. Girlanda, "Nonequilibrium Langevin approach to quantum optics in semiconductor microcavities," Phys. Rev. B 77, 035433 (2008).

[76] O. Di Stefano, S. Savasta, and R. Girlanda, "Mode expansion and photon operators in dispersive and absorbing dielectrics," J. Mod. Opt. 48, 67-84 (2001).

[77] C. W. Gardiner and M. J. Collett, "Input and output in damped quantum systems: quantum stochastic differential equations and the master equation," Phys. Rev. A 31, 3761-3774 (1985).
[78] S. Savasta, O. Di Stefano, and R. Girlanda, "Light quantization for arbitrary scattering systems," Phys. Rev. A 65, 043801 (2002).

[79] O. Di Stefano, A. F. Kockum, A. Ridolfo, S. Savasta, and F. Nori, "Photodetection probability in quantum systems with arbitrarily strong light-matter interaction," Sci. Rep. 8, 17825 (2018).

[80] A.D. O'Connell, M. Hofheinz, M. Ansmann, R.C. Bialczak, M. Lenander, E. Lucero, M. Neeley, D. Sank, H. Wang, M. Weides, J. Wenner, J.M. Martinis, and A.N. Cleland, "Quantum ground state and single-phonon control of a mechanical resonator," Nature 464, 697-703 (2010).

[81] F. Rouxinol, Y. Hao, F. Brito, A. O. Caldeira, E. K. Irish, and M. D. La Haye, "Measurements of nanoresonatorqubit interactions in a hybrid quantum electromechanical system," Nanotechnology 27, 364003 (2016).

[82] W. Qin, V. Macrì, A. Miranowicz, S. Savasta, and F. Nori, "Experimentally Feasible Dynamical Casimir Effect in Parametrically Amplified Cavity Optomechanics," arXiv:1902.04216 (2019). 